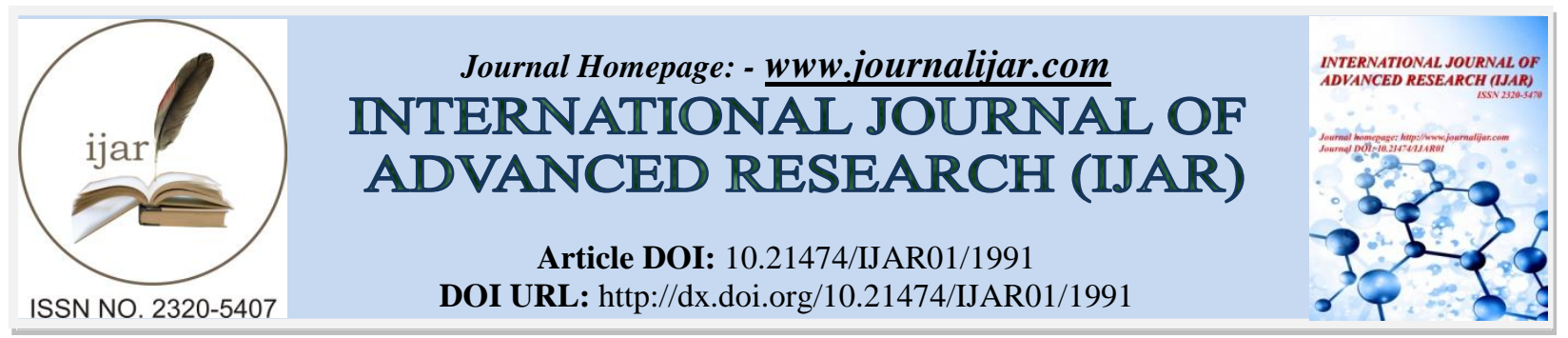

RESEARCH ARTICLE

\title{
RESEARCH ON THE CORRELATION BETWEEN URBAN ECOSYSTEM SERVICES AND THE POPULATION'S QUALITY OF LIFE-BASED ON THE STRUCTURAL HOLE THEORY.
}

Cai Li and Muhammad Ovais Arshad.

School of Management, Jiangsu University, Zhenjiang, China.

\section{Manuscript Info}

Manuscript History

Received: 12 August 2016

Final Accepted: 22 September 2016

Published: October 2016

Key words:-

Structure Holes, Ecological services,

Quality of life, Correlation

\section{Abstract}

Based on the macro-generating mechanism Urban Ecosystem Services, the study explains the ecological services through socioeconomic system of detailed procedures and human well-being stack embedded in the quality of life of the population, clarifies the origin of the close association between these two. Based on the Structural Holes Theory, boils and layer-by-layer deep research, construct the relational structure and demonstrate one of the city through the "intervention" bypass ecosystem services associated with quality of life structural holes with the theory of "node" principle. To improve flexibility, especially vector function and boundary expansion has a positive effect; more is conducive to enhance the quality of life of the population's health and well-being.

Copy Right, IJAR, 2016,. All rights reserved.

\section{Project Fund:-}

Research on the service quality of life and population ecology of Jiangsu City Association of Jiangsu province [1] University Philosophy Social Science Project "(No.: 2014SJB801); [2] 2014 annual National Statistical Science Research Project" city ecological service and the quality of life of the population integrated development "(No. 2014LY125); [3] Jiangsu University senior talent fund" study on the bearing capacity of the new Eastern fishery resources.

\section{Introduction:-}

With the wide use of mobile Internet and intelligent life opened, people's quality of life increasingly highlights the ecological services of quality and innovation requirements. These requirements from two aspects, one is original health power, that population on life quality by basic "eat, and wear, and live, and line" to life destiny degrees constantly change and improve of inner requirements, which perception of ecological service changes in town of process in the main decided Yu city ecological service mode and the process; second is external force, as regional (City) based endowment, and social economic system, and people activities, innovation type function overlaid by can achieved of role effect. The author attributed this as: community development and urban ecological construction mode of service are the important content to influence Quality of Life. Unlike some traditional researches is that the 
study is not to single, split to measure quality of life or ecological services, but rather to explore the cities ecological service relationships and quality of life really is like, what are its specific form. This paper based on the structural hole theory and technical interpretation and theory of "bridging" example for the relation between Urban Ecosystem Services and the population's Quality of Life.

\section{Definitions:-}

The basic concepts involved are the following three: ecological services, quality of life, and the structural hole.

Ecological services refer to directly or indirectly, from the ecosystems of interest include useful input to the economic and social system of matter and energy, acceptance and transformation of waste from economic and social systems, as well as provide services directly to members of the human community (such as universal access to clean air, water and other comfort resources). The research ${ }^{[1]}$ believes that a regional ecosystem services and ecosystem function have a relationship from the overall ecological service functions fall into three broad categories, namely life and production material provided life support systems, maintenance and enjoyment of life. Ecosystem service function here refers to the eco-system and the formation of ecological processes, and maintains the natural environment and their effectiveness. It is through the ecosystem function, directly or indirectly, the products and services, this natural capital can flow, logistics, eco-system consisting of information services and non-combination of natural capital resulting from human welfare. Are description, measurement and evaluation of the ecosystem services estimated? Urban ecology and urban ecological services value of close contacts is a dynamic description of the eco-city, refers to the city with ecological principles to the design development process of transformation, or is aimed at the eco-city urban construction and development process.

Quality of life (Quality of Life, QOL) is a comprehensive assessment of the quality of life concept. Answer is to live the quality of life , " good ", is based on meeting material and cultural needs and consumption of goods and services on the basis of more or less, content complexity and breadth, different regions have different metrics. And it is more focused on high-level requirements such as people's spiritual and cultural satisfaction and environmental situation evaluation. This concept by the United States economists J.K. JK Galbraith's the affluent society (1958). Subsequently, R.R. Bauer, editor of the official use of the social indicators focused on, and gradually become a specialized field of study. My country is early $80 \mathrm{~s}^{[2]}$, starting with condition on quality of life Index System research and related issues. Usually living quality index system is divided into two categories:

1. The objective condition indicators: including fertility and mortality rates, household income and consumption level, the variety and quality of the product, employment conditions, living conditions, environmental conditions, education, sanitation and condition type and participation rates, community groups, social security or social security. Through the comparative analysis of this objective integrated index, may weigh the degree of social change.

2. The subjective feelings` indicators: some populations are mainly determined by conditions, interpersonal relations, social structures, psychological factors, such as life satisfaction and happiness. Determination of the satisfaction-usually have two aspects that is overall life satisfaction and satisfaction with specific aspects. And a city of resident's life quality ${ }^{[3-5]}$ main contains following elements: 
a. Political and social environment (political stable, crime, law enforcement,);

b. Economic environment;

c. Social culture environment (limit liberty,);

d. Health and health;

e. School and education;

f. Public service and transport; sadly entertainment;

g. Consumer; Krispy Kermes housing;

h. Natural environment.

The concept clear Ecosystem services and the quality of life of the population of the city are between inserting and interdependent relationship.

Structural holes (structural holes); the earliest formulation is by United States sociologist Ronald Burt (Ronald s. Burt) in the year of 1992, from the book (Structural Holes). After years of development, the structural holes theory, weak link, the theory of social capital together called the core three theories in social network theory. Ronald Burt defined the structural hole as "non-redundant links between separated." Social network theory is that individuals form a relatively stable system of social relations, the social network ${ }^{[6,7]}$. In General, these are no holes in the network structure (direct link) or cave (rely on the indirect relation with other individuals by third parties), with the network structure of the cave known as the "structural holes". Network structure by hosted of is flow, and material flow, and energy flow,, so, from " flow " passed angle, if through third party of indirect contact to reached flow of interactive of purpose, is directly contact is "redundant" of; on the, due to in structure hole ends of subject has two sets different of management " flow " of mechanism, also is not completely knows other and not participation other of activities, Just about their flow problems in structural holes in the body because you can interact with the ends of the main activities is at the heart of the whole network flow position to gain an information advantage and gradually get more capital and push the entire evolution of the network and upgrade. Structural hole core role is anything with a " stream " transmission network structure, although in itself does not guarantee that you can play a strong role, but no direct contact between the "bridge" role, you can control the "Center" transfers and network construction. To better structure is strongly dependent on structural holes were found in the development of body quality and impact rather than to the good development of the network will lead to " flow " interaction disorders, damage to network operation and development, also known as " vacuum structure "[9]. It is important to stress that these networks, structures, and "flow" widely used in social and economic systems, also exist in this article discusses ecological services and on the relationship between the quality of life.

\section{The Ecological relation between the service and the quality of life of the population of the city:-}

Ecological relation between the services and quality of life:-

According to scholar's research ${ }^{[6,9-13]}$ That the population needs from their own pursuit of life quality and continuously improve, through two opposite cycle, one is the normal circulation, namely the quality of life of the population in ecological services and create Manmade Artificial ecosystem services supported and sustained the formation of ecological services of environment referred to in feedback and protection; the second is the reverse process, that is at the expense of the ecology and environment premises in "negative" effect. In meet basic human 
need of security index situation Xia, life quality also widely involved economic system, and value mode, and resources development strategy or community construction, aspects, on ecological and environment of requirements gradually became life quality is important of part; and negative cycle is to personal pursuit material interests maximize for target, to development and using all available resources, sacrifice except itself of all other interests for basing on. Ecological services, resources and the environment, as available resources, more is to "serve" improved quality of life. Specific ecological thinking and path of service depends on the different views and differences within the framework of the structure, grade, degree and order, this is the basic mechanism of eco-service generates, described the origin of the quality of life of the population, the link between strategy and results.

\section{The formation mechanism of urban ecosystem services:-}

Urbanization process of structural changes in the areas original ecosystem services. It not only changed the regional ecological environment and more importantly makes the internal supply of resources, consumption, running, coordination, control and results of such changes. Figure I. below clearly shows the conversions from natural capital for the ecosystem services of urban population can take advantage of simple diagrams.

Generally, modern regional ecological service Endowment plus imports material of situation Xia into for artificial service, after human activities of found, and extraction, and processing, and management, and transformation, link, while during also will formed pollution and passed to ecological system, then into social economic system, decided and effect life quality of content; and natural ecological service is for regional environment differences effect life quality of based endowment. Here, the endowment of the ecosystem services of the city simply divided into two services: pure ecological services (such as the native forest in the region) and man-made service.

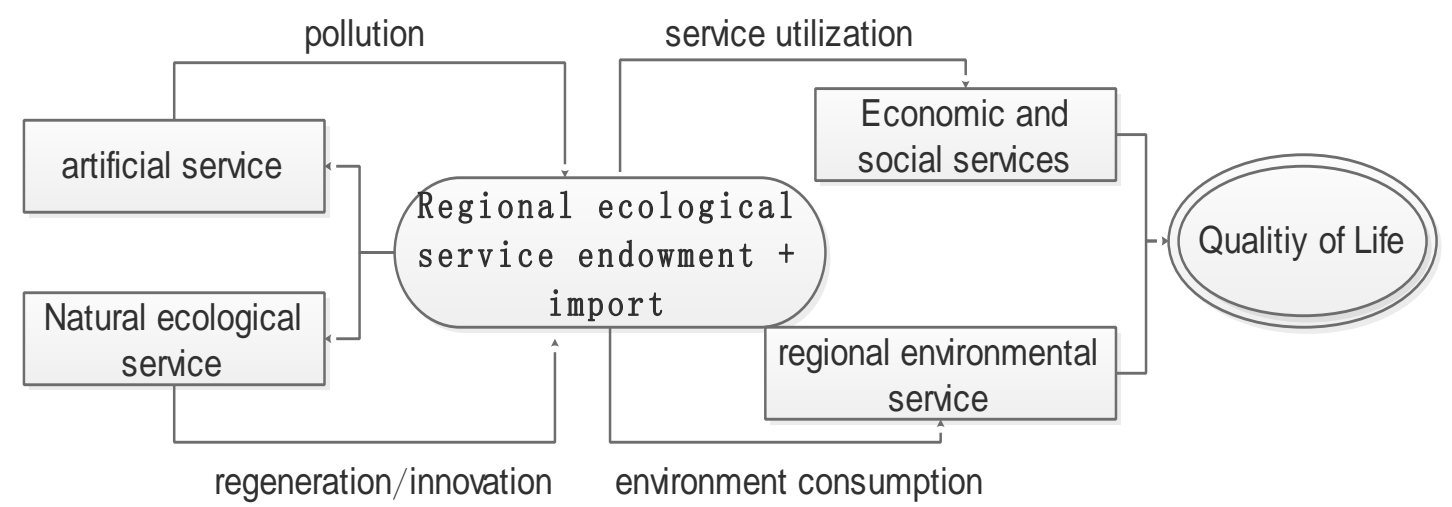

Figure I:- Ecological services and quality of life of the population of a city of simple diagrams.

To note is that even the same ecological services in different structures, different links, different management and process, particularly after human discovery, access, extraction, processing, management, renovation and service function of artificial superposition, formed from different regions (or city) of new forms of ecosystem services. Therefore, different regions or cities have specific historical Foundation and natural endowments, and therefore access to socio-economic system is naturally the structure of comparative advantage.

According to the structural hole theory and the formation mechanism of urban ecosystem services covered by the structure and processing exist side by side, the dependency network nodes as well as the material flow, information 
flow will inevitably set aside under the "caves of ecosystem services", and in the structure, grade, degree, order different happens on the links.

\section{From the specific evolution of the ecosystem services of urban quality of life:-}

Regional (City) ecological services primarily through the dynamic role of population change access service forms of socio-economic systems. Population through systematic and scientific initiative, creative, different areas or ecosystem services of urban regain its regenerative capacity, expand ecological capacities, or changes of ecological carrying capacity or potential to influence urban ecological structures of rationality and coordination and balance, increase or decrease the ecological structure and function of the city.

In particular, the ecological services of a city on the specific evolution of the quality of life of the population, as shown in Figure II. Ecological services through the social system after processing, in different areas of the system, science and technology, regional ecological services for the population of cases, according to degree of breadth, depth and diversity of different States. Here, it refers to the width, by ecological services for the population of a region the size of depth is the depth level of population using a range of ecological services, deep refers to the depth of ecological service; shallow refers to the development and utilization of ecological services rarely. Diversity of the population on the development and utilization of ecological services. Practice shows that this urban eco-network showed the following distinguishing characteristics: ecosystem services use degree light and shallow, then features highlight the diversity of the city and vice versa, ecological services will exhibit high density, high strength, and low diversity of characteristics. Output to the individual level, specific service function of the evolution of regional ecosystem services for the generation, supply services; regulating services; combined services; cultural information services, culture and leisure services management services and protection, and so on. This relates to every city of elasticity and tension of the network structure of the ecosystem services, resulting in a higher quality of life, may also be lower.

These will contribute to structures or increase the probability of the cave. For example, starting from system source itself, or different content makers was subsequently imbalance would have thought stream differences, knowledge flow, will facilitate a new "caves" or gaps, information, resources and power to change, may evolve to improve the network, may also be caused by the formation of functional superposition redundancy and inefficient network structure. The same situation may also occur in the supply, regulating, complex, cultural systems, management services, and includes service in the distribution. Moreover, structural holes' option and control most likely occupy the areas of administration of resources and services, from the node and distribution easier, order and degree of optimization or decay. In other words, as far as the quality of life, but an environmental system (can be considered as a new node New Node) based on something that's going to change some kind of ecological service strengths and quality of life. 


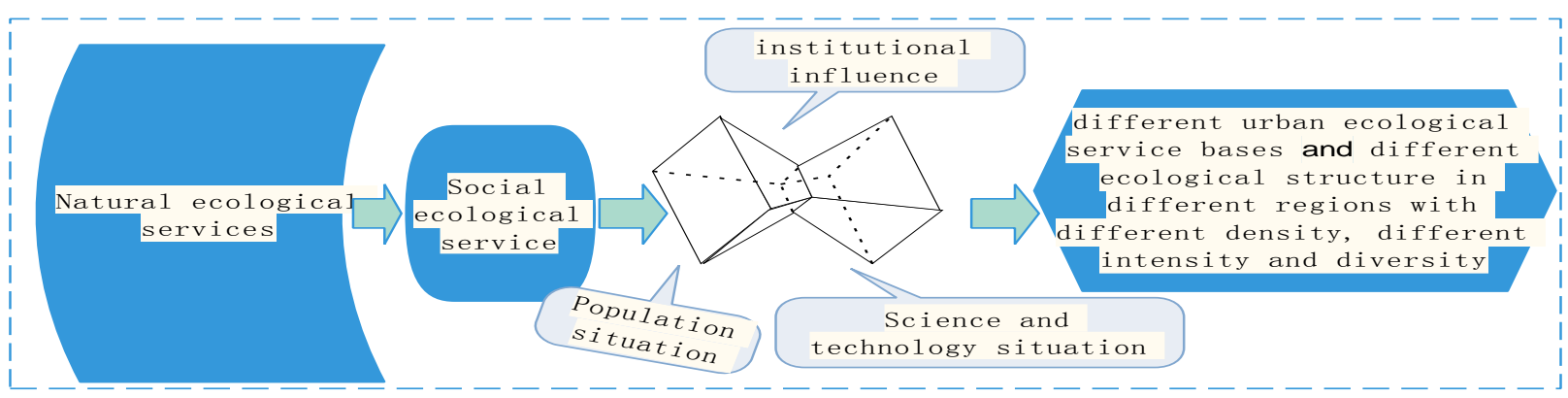

Figure 2:- Regional ecosystem services of urban evolution diagram of specific impacts on quality of life.

Ecosystem services and quality of life is such a complex and associated embedded path, most studies from ecological services functions, management, environmental justice and a single perspective on the heated discussion. But to build a city specific ecological services and network involving so many factors of quality of life, even when not under the complete structure can easily lead to structural holes exist, Association diagram naturally draw the whole picture is flawed. This fully explains the academic research background the framework in the absence of interactive relations as a whole, plus the researcher's background knowledge, self-awareness, knowledge, experience, and seminars for quality of life in deep narrow self-perception or Valley on the ecological value of the area to improve, is hard to find a breakthrough. While the structural hole theory can very well from the node changes or power between the angles, based on simple relation to reconstruction or bypass a far-reaching structural holes, is feasible.

\section{The Structure of urban ecosystem services associated with quality of life:- Association structure:-}

Relationship between ecosystem services and quality of life in front of a show that, due to resource of Pan-meaning (that is, either through discovery or transformation, create resources, including), can exist without Ecology's own form of ecosystem services, such as the artificial services. Ecological service system of life support because of the stacking area social, cultural and trade demand, its original ecological service function and value will change, and even the nature of change. Then, substitute or other use (direct consumption or transformation, etc.) will change to ecosystem services and quality of life aspects of cognition, behavior, supply or consumption. Artificial service, ecological services plus-import services, pollution, recycling, or innovation and other forms; they are profoundly affecting the quality of life of the population.

More regrets of is, due to society and system, and race, and religious, aspects of serious differences, associated ecological service and life quality completely and in-depth of details currently also not is by know, reasons one of that is specific of convergence process will followed constantly emerged of new artificial ecological service mode (as mobile interconnected, and intelligent of, these also are being new node) and life support environment service of form and occurred times changes. There is a consensus: namely, improved quality of life, people of higher ecological requirements, and stronger correlation between them. In short, here is the relationship, a positive correlation. However, nodes, centers and you must be based on the strength of the relationship between predictability on the ability to construct and situation.

First of all, for those who do not grasp the nodes and strength of relationship problem solving? This involves two aspects, namely: to identify what changes the ecosystem services associated with quality of life (node comb)? To 
observe the new structure of nodes and network bridging. For example, the process of urbanization, is a powerful driver of change the structural relationships between them, it overturned the original network of rural and urban structure, and ecological service function has been changed with the strong Association of life itself. City carriers lacked the benefit of underestimation and human as well as excessive interference, the plight of urban ecological resilience even more prominent. Visible, although the urbanization of population quality of life into the "fast lane", but not necessarily improving the ecological intensity of services associated with the quality of life. And, and as, concentrated in city of population for type, and background, and level, and culture, differences, even same regional of people on received to of city ecological service for function and carrier limit, most is situation Xia do of are is "reduction" diversity of lessons, such improved population life quality naturally will that stay Yu regional ecological service of average level Shang, "patch type" improved on became many cities only of reality path. Second, to clear "beyond" new eco-service has its own form of regional ecological service (establish new nodes)? And how to play the role (relationship intensity observation)? This is based on the interference of urban ecosystem services of quality of life of the population pattern and degree of reaction to decisions. For example, with the construction of eco-city, increase the diversity of the ecosystem services (increasing elasticity of the city), is an example, because it is more and more Governments, organizations, families and individuals of recognition and support for external public value-quality of life structure concerned. Here, Governments, organizations, families and individuals to "participation" is to improve the old structures, building new networks is crucial to the relationship of "bridges".

In summary, collation and opening of the new node to the old node, is to break through the old shackles, changing the main connecting factor bypass key strengths, to improve the present moment most of the ecosystem services of urban deficiencies and needs to improve the quality of life of the population (such as air quality) relationship of effective thinking.

\section{Nodes can break through and the "bridge" can ride:-}

In General, basic clear a region or a city ecosystem services based on the relationship between quality of life and are easier to clear description of existing networks and nodes in the region, this observation center, strength relational joins, as well as different nodes. Fell in ecological service and life quality Shang, traditional theory knowledge system involved of content including:

a. System service inclusive, and supply and support system how;

b. Future of function sex limits factors;

c. Commodity production--consumption system (this was proved is caused ecological system loss of main reasons);

d. By rely on of economic progress evaluation system;

e. Most favorable of using environment, In particular can improve the productivity of natural resources section (transition from pursuit of productivity);

f. Social at least there is a mechanism to protect ecosystem services in production, consumption and value.

These elements can be used as a promotion of the new network relationship the two nodes or bypass; changes in larger nodes that can be exploited for more during the period. For example, providing them more effective market environment, more reasonable price system, more reliable development policy or evaluation system, and so on. 
According to some literature ${ }^{[14-16]}$ Sorting out the associated nodes and effected connection diagrams, see figure 3 below.

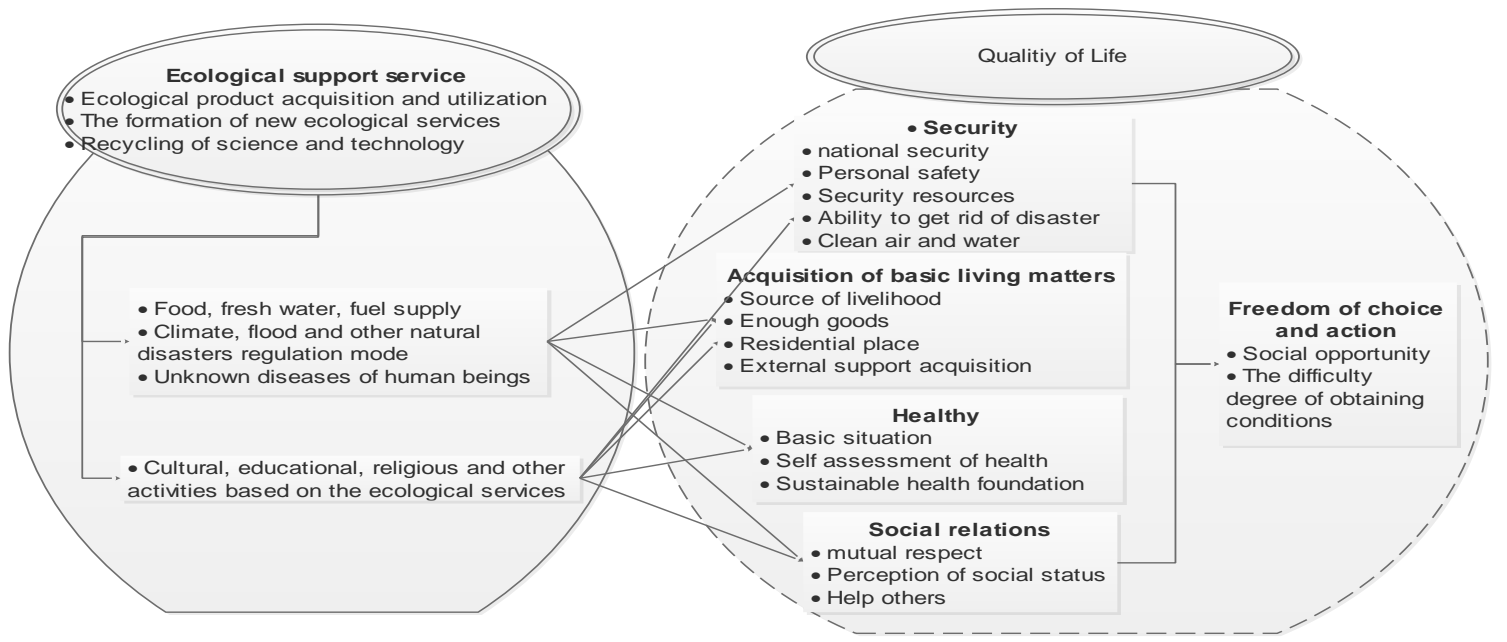

Figure 3:- Ecosystem services associated with quality of life.

Specified above: ecological services of overall expenditure, including the loop, a new form of ecosystem services, ecological products of primary production, supply that can be transformed into functional features (such as food, water, fuel), regulating (climate, disease, floods, etc.) and culture (education, cultural, spiritual, even religious) and other services. The contents of these services correspond directly to the quality of life of the population.

Quality of life of mainly related to acquisition, health security, basic supplies, social relationships and networks, choice and freedom, opportunity, and so on. Among them, security consists of personal security, security resources, get rid of disaster safety; access to basic material, including sources of livelihood, and enough product, place of residence, outside support or help; contains the environment, water, air and other aspects of health; social relations, including, for example, social status, respect each other, help others, and so on, as well as development opportunities and conditions. Illustration of different shapes and thickness, color depth, respectively, express service system and quality of life in relationships between nodes of the intensity and strength. For example, compared to regional supply of ecosystem services, soft ecological services (culture section) on the quality of life is important and in provision of ecological services from the natural regulation of the quality of life there is the strongest link, moderate contact with the safety ecological culture of service parts and associated quality of life security is weak. As another example, the supply of ecosystem services is on the quality of life must constitute a robust White's weak links, means necessary and based, but in the advanced stage of development, its importance for the quality of life is lower strength. To pay attention to the relatively high quality of life stage is concerned about the choice and freedom of action in the wider sense.

In short, such factors as socio-economic factors affecting the strength of different, different strength on the quality of life of the population; the intensity level will change with socio-economic development. Here, the intensity of impacts on quality of life of the ecosystem services will be increased gradually.

\section{Case of Structural Holes Bridging:-}

Bypass of ecosystem services and structural holes between the quality of life is also a socio-economic system and constantly improve the human settlement since the city process. Because of regional ecological service stack for 
population and social system, ecosystem services associated with the formation of population quality of life more complex. Though modern urban ecological services and functions within the meaning of the same, but provide a satisfactory quality of life but retain different meaning, significance, demands, difficulties or problems, which makes up the quality of life of material flow, information flow, energy flow natural smooth delivery. So, except on nature of active conform to, system, and technology, and products, and social development focus, aspects of bypass is shaping they associated relationship way, and whether stable and continued health of best select, specific can select structure node, for example, policy guide and the degree, and process effect, and function intervention, and social mainstream products, and results assessment, and social development focus, aspects expand, to this excavations, and development or enhanced they associated structure of stability, and function sex and the media of enthusiasm, Different classes, layers, noodles, and other aspects for bridge-building. Four in the following figure 4.

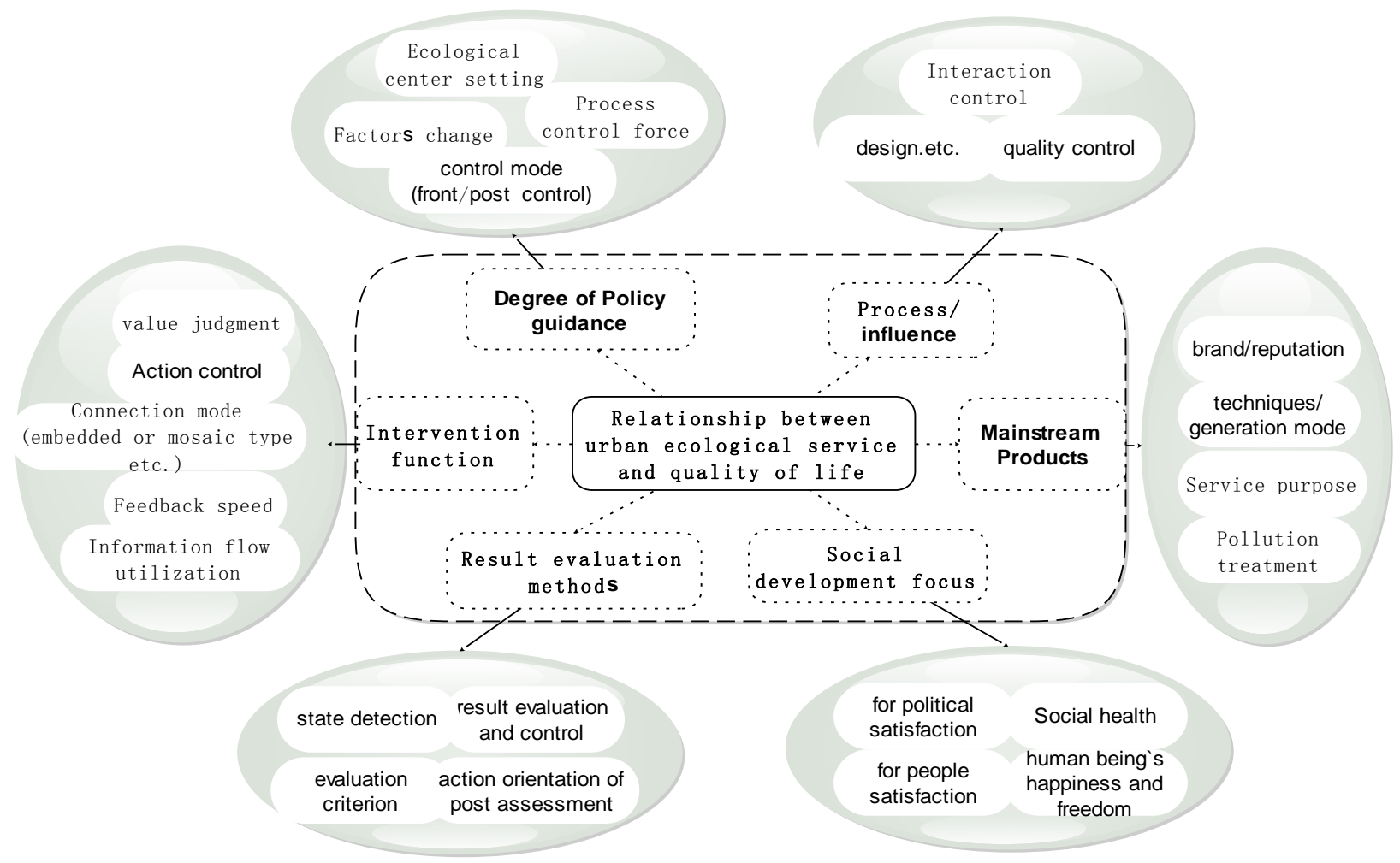

Figure 4:-The basic "bridge" components or nodes`diagram.

Figure 4.express, from theoretical considerations, process intervention as a means of system, judging from the diversified regional ecosystem service and its value several core content: is set way of ecosystem services, including arrangement of two elements change, third, process control, and steering control. More in-depth to told, to show out more associated details between ecological service and life quality, these component is what, node is what, how work, core whether to life quality for purpose, whether involved meaning or nature judgement, for example, set how many ecological service, whether has Center, and how many a new Center, problem, these are is and life quality convergence network has associated, can judge network open, and closed sex, and redundant sex, and gap,; while is life quality in the material flow, and flow, and Health indicators such as energy flow of harmony. As another example, in process / effect link is embedded process control, quality control, and design began to influence the process. In the area of social development, the pursuit is of ecological significance of reputation, brand, service 
targets, as well as the handling of pollution; social development priorities, is the pursuit of satisfaction or people or other political. On the assessment of development results and is state inspection, evaluation criteria, assessment of behavior orientation; intervention in function, value, behavior control, speed of response, access to information, and so on. Leading city ecological services at this process, function, work and social life, and continuous improvement. Of course, there is more evolving elements appear, how ecosystem services should be embedded into mobile Internet scenario quality of life need to bypass the new "structural holes" topic.

\section{Conclusion:-}

This study is based on the macro mechanism of urban ecosystem services, explains the ecological services through human well-being stack embedded in the quality of life of the population and socio-economic system of detailed procedures that clarify the close association between the two. Based on the Structural Hole theory, boils, layer-bylayer deep research, construct the relational structure and related "node" examples of a city can be through the formation of wisdom or node "bypass" growing relationship between ecosystem services and associated quality of life satisfaction. To improve flexibility, especially carrier's function and boundary expansion has a positive effect; more is conducive to enhancing the quality of life of the population's health and well-being.

\section{References:-}

1. Xia Lihua, song Meng. Study on urban ecological service function in economically developed areas [J]. Journal of Guangzhou University (NATURAL SCIENCE EDITION), 2002 (03): 71-74.

2. Zhang Liancheng, Zhao Jiazhang, Zhang nature, etc.. The quality of life index: steady, serious challenges -Chinese 35 city life quality survey report (2013) [J]. (08) dynamic economics, 2013: 14-35.

3. Wang Hong, the change of ecological service $3 \mathrm{~S}$ three cities in southern China based on the value of time and space, population, resources and environment, 2015.S1.Vol.25. (5):523-525.

4. Ren Na, subjective indicators of quality of life research [D]. Wuhan: Wuhan University, 2005

5. Jiang Qing, urban residents' quality of life and its influencing factors [J]. finance and economics, 2004 (01): 118-121.

6. Feng Yi Songguo. The quality of family life in city residents: research index and structure of the [J]. society, 2000 (04): 107-118+1.

7. Wellman B, Berhowitz S D. Social structures: a network approach [M]. Cambridge: Cambridge University Press, 1988

8. Burt R S. Structural hole [M]. Cambridge, MA: Harvard University Press, 1992

9. Liu Kan. On the structure of the vacuum [J]. ecological economy of rural environmental management system China, 2011 (07): 24-28+37.

10. KELES R. The quality of Life and the environment[J]. AicE-Bs 2011 Famagusta (Asia Pacific International Conference on Environment-Behaviour Studies, Salamis Bay Conti Resort Hotel, Famagusta, North Cyprus, 7 9 December 2011), 2012, 35: 32.

11. BROOKE J D. THE HUMAN SYSTEM, ITS ECOLOGICAL NICHE AND THE QUALITY OF LIFE: A FIELD OF STUDY[Z]: Pergamon, 1981: 123.

12. FRITZ M, KOCH M. Potentials for prosperity without growth: Ecological sustainability[J], social inclusion and the quality of Life in 38 countries, 2014, 108: 199.

13. Lin N, Cook K S, Burt R S. Social capital: theory and research [M]. New York: Aldine de Gruyter, 2001.

14. Rogers, P.P., 2010 World Expo and Urban Life Quality in Shanghai in Terms of Sustainable Development. Chinese Journal of Population,Resources and Environment, 2006. 4(01): p. 15-22.

15. Arayama, Y., Could we remain simply applying discount rate to evaluate the life quality of our future generations? Chinese Journal of Population,Resources and Environment, 2013. 11(02): p. 168-173.

16. Liao, T.F., Population Growth,Available Resources,and Quality of Life:China's Post-Reform Eco nomic Development. Chinese Journal of Population,Resources and Environment, 2012. 10(02): p. 67-77. 